\title{
Transfer Learning for Rapid Re-calibration of a Myoelectric Prosthesis after Electrode Shift
}

\author{
Cosima Prahm Benjamin Paassen Alexander Schulz \\ Barbara Hammer Oskar Aszmann *†
}

October 23, 2016

\begin{abstract}
For decades, researchers have attempted to provide patients with an intuitive method to control upper limb prostheses, enabling them to manipulate multiple degrees of freedom continuously and simultaneously using only simple myoelectric signals. However, such controlling schemes are still highly vulnerable to disturbances in the myoelectric signal, due to electrode shifts, posture changes, sweat, fatigue etc. Recent research has demonstrated that such robustness problems can be alleviated by rapid re-calibration of the prosthesis once a day, using only very small amounts of training data (less than one minute of training time). In this contribution, we propose such a re-calibration scheme for a pattern recognition controller based on transfer learning. In a pilot study with able-bodied subjects we demonstrate that high controller accuracy can be re-obtained after strong electrode shift, even for simultaneous movements in multiple degrees of freedom.
\end{abstract}

*Funding by the DFG under grant numbers HA2719/6-2 and HA2719/7-1, the CITEC center of excellence (EXC 277), as well as the Christian Doppler Research Foundation of the Austrian Federal Ministry of Science, Research and Economy is gratefully acknowledged.

${ }^{\dagger}$ Cosima Prahm (cosima.prahm@meduniwien.ac.at) and Oskar Aszmann are with the Christian Doppler Laboratory for Restoration of Extremity Function of the Medical University of Vienna, Austria.

$\ddagger$ Benjamin Paassen, Alexander Schulz and Barbara Hammer are with the Theoretical Computer Science group of CITEC Bielefeld, Germany. 
Preprint of the publication [9. The original can be found at DOI:10.1007/978-3-319-46669-9_28

\section{INTRODUCTION}

An intuitive and natural control of upper limb prostheses enables patients to carry out everyday tasks faster and easier, significantly improving quality of life [2]. Currently, the only viable option to achieve such a control is the decoding of surface myoelectric (EMG) signals via machine learning techniques, such as pattern recognition or classification, to extract the user's motion intent and drive the artificial limb accordingly [2]. However, machine learning algorithms require signals corresponding to the same intended motion to be constant over time, while in practice the myoelectric signal is vulnerable to disturbances, such as electrode shifts, posture changes, sweat, fatigue, etc. [5, 12. As of today, these problems with respect to robustness are too severe to apply machine-learning algorithms as part of a commercial product [2]. Several approaches have been suggested to improve the robustness, such as implanted EMG electrodes instead of surface electrodes [4], high-density EMG surface electrode-grids [6, 11], more sophisticated signal pre-processing (feature extraction) [5], and post-hoc error detection in the decisions of the machine learning algorithm [1. However, a method that achieves robust control using only a moderate amount of surface EMG electrodes is still lacking [2]. Therefore, recent research has suggested an alternative approach, namely developing a re-calibration scheme that permits users to regain the high accuracy obtained under lab conditions, using only minimal training information (less than 1 minute of recording time) [12. While promising, the current state-of-the-art is not yet able to fully regain the recognition accuracy obtained under lab conditions and does not yet allow simultaneous movements. In this contribution, we extend the re-calibration approach by applying a novel transfer learning technique to infer the sensor disturbance caused by a shift of the electrodes and adjust the incoming signal accordingly. We evaluate this approach in a pilot study with four able-bodied subjects executing simultaneous motions in multiple degrees of freedom.

\section{MATERIALS AND METHODS}

\section{$2.1 \quad$ Transfer Learning}

Transfer learning is concerned with solving a given task by transferring knowledge from a related task [8]. In this case, we attempt to control an upper limb prosthesis under disturbance by using knowledge obtained in a disturbance-free lab setting. Under the assumption that the disturbance can be described by a linear transformation matrix $\mathcal{I}$, our aim is to find the in- 


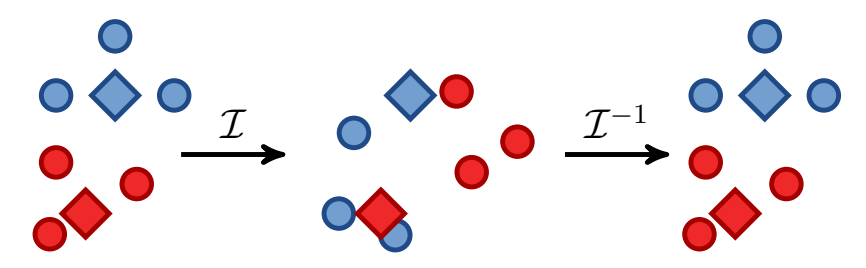

Figure 1: A schematic view on the transfer learning approach. Left: A machine learning model (diamonds) is fitted to data (circles). Middle: A disturbance $\mathcal{I}$ causes a mismatch between model and data. Right: By learning and applying the inverse disturbance $\mathcal{I}^{-1}$ the data can be cleaned again.

verse $\mathcal{I}^{-1}$, such that the disturbance can be removed from the data, and the originally learned model can be applied again (see Fig. 1).

In this contribution, we exemplarily apply this approach to a Generalized Matrix Learning Vector Quantization (GMLVQ) classifier, which describes the data by representative prototypes (diamonds in Fig. 1) and infers the intended motion of a patient by assigning the motion corresponding to the closest prototype to the current myoelectric signal [10]. We infer the matrix $\mathcal{I}^{-1}$ via gradient descent on the GMLVQ cost function [10. GMLVQ itself as well as the gradient descent for transfer learning are on-line algorithms with linear-time comlexity, which makes them applicable in real time-settings.

\subsection{Setup}

Four able-bodied subjects were instructed to execute six movements in three degrees of freedom (pronation and supination, flexion and extension, as well as hand open and hand close) as well as the twelve pairwise combinations of these movements and resting, resulting in 19 different movements overall. Each movement was executed for five seconds, followed by two seconds of rest. Muscle activity was recorded at $1000 \mathrm{~Hz}$ sampling rate with an eight channel Ottobock Healthcare electrode array (13E200) attached around the forearm. To simulate doffing and donning of a prosthesis, the electrodes were shifted transversally by $8 \mathrm{~mm}$, as in [12], and all movements were recorded once more. Transversal shift was applied as it has been shown to be more challenging than longitudinal shift [6]. As signal preprocessing, a $90 \mathrm{~Hz}$ to $450 \mathrm{~Hz}$ band pass filter was applied. On windows of $100 \mathrm{~ms}$ with $50 \mathrm{~ms}$ overlap the 17 standard features offered by BioPatRec [7] were computed, in addition to the log-variance as suggested by [3]. For each subject, four classifier models were trained, one to distinguish the resting state from actual movement, and one to recognize motion in each degree of freedom, permitting the recognition of simultaneous movements. As part of the training, metric learning/feature 
Preprint of the publication [9. The original can be found at DOI:10.1007/978-3-319-46669-9_28

\begin{tabular}{l|ccc} 
degree of freedom & original & shift & re-calibrated \\
\hline pronation/supination & $0.6 \%(0.3 \%)$ & $36.2 \%(15.4 \%)$ & $3.6 \%(2.5 \%)$ \\
flexion/extension & $0.5 \%(0.5 \%)$ & $10.9 \%(3.7 \%)$ & $1.3 \%(0.9 \%)$ \\
open/close & $0.7 \%(0.4 \%)$ & $29.1 \%(6.4 \%)$ & $3.8 \%(1.0 \%)$
\end{tabular}

Table 1: The average recognition error (in percent) across subjects. The standard deviation across subjects is given in brackets. Rows mark the degree of freedom, columns the experimental conditions.

selection was applied, as suggested in [10]. Training was done in a 10-fold cross-validation. In each fold, we used only five folds (50\%) of the data to learn the inverse disturbance matrix $\mathcal{I}^{-1}$ and evaluated the recognition accuracy on the remaining single movements as well as the simultaneous movements. This work was approved by the ethics commitee of the Medical University of Vienna (\#1301/2015).

\section{RESULTS}

The experimental results are shown in Table 1. For all subjects, the initial accuracy of the classification model is high (below 1\% error). After electrode shift, the recognition accuracy degrades drastically, with $10 \%$ to $36 \%$ error on average, depending on the motion performed. Transfer learning decreases the classification error again, achieving accuracy of $95 \%$ and more. The difference between the accuracy after electrode shift without transfer learning and with transfer learning is highly significant for all subjects and motions $(p<.01$ using the Wilcoxon rank sum test and Bonferroni correction).

\section{DISCUSSION}

As expected, electrode shift leads to severe degradation in recognition performance. Fortunately, our proposed transfer learning scheme significantly improves the performance again to near-perfect accuracy, outperforming the previously reported results by [12. These results hold even for simultaneous movements in multiple degrees of freedom. 
Preprint of the publication [9. The original can be found at DOI:10.1007/978-3-319-46669-9_28

\section{CONCLUSION}

Our pilot-study indicates that transfer learning is a promising scheme to rapidly re-calibrate a pattern recognition system after performance degradation due to signal disturbances (e.g. electrode shift). With only 30 seconds of recorded data, recognition accuracy can be significantly increased, even for simultaneous movements in multiple degrees of freedom. Directions for further research include transfer learning for different machine learning models, other sources of disturbance than than electrode shift, in particular posture changes, and more extensive experimenal evaluations, including amputees as test subjects. Such research might lead to prostheses, which are adaptive to everday disturbances with only minimal effort required by the patient.

\section{References}

[1] S. Amsüss, P. M. Goebel, N. Jiang, B. Graimann, L. Paredes, and D. Farina. Self-correcting pattern recognition system of surface emg signals for upper limb prosthesis control. IEEE Transactions on Biomedical Engineering, 61(4):1167-1176, April 2014.

[2] D. Farina, N. Jiang, H. Rehbaum, A. Holobar, B. Graimann, H. Dietl, and O. C. Aszmann. The extraction of neural information from the surface emg for the control of upper-limb prostheses: Emerging avenues and challenges. IEEE Transactions on Neural Systems and Rehabilitation Engineering, 22(4):797-809, July 2014.

[3] J. M. Hahne, F. Biebmann, N. Jiang, H. Rehbaum, D. Farina, F. C. Meinecke, K.-R. Müller, and L. C. Parra. Linear and nonlinear regression techniques for simultaneous and proportional myoelectric control. IEEE Transactions on Neural Systems and Rehabilitation Engineering, 22(2):269-279, 2014.

[4] J. M. Hahne, D. Farina, N. Jiang, and D. Liebetanz. A novel percutaneous electrode implant for improving robustness in advanced myoelectric control. Frontiers in Neuroscience, 10(114), 2016.

[5] R. N. Khushaba, M. Takruri, J. V. Miro, and S. Kodagoda. Towards limb position invariant myoelectric pattern recognition using time-dependent spectral features. Neural Networks, 55:42-58, 2014.

[6] S. Muceli, N. Jiang, and D. Farina. Extracting signals robust to electrode number and shift for online simultaneous and proportional myoelectric 
Preprint of the publication [9. The original can be found at DOI:10.1007/978-3-319-46669-9_28

control by factorization algorithms. IEEE Transactions on Neural Systems and Rehabilitation Engineering, 22(3):623-633, May 2014.

[7] M. Ortiz-Catalan, R. Brånemark, and B. Håkansson. Biopatrec: A modular research platform for the control of artificial limbs based on pattern recognition algorithms. Source Code for Biology and Medicine, 8(1):1-18, 2013.

[8] S. J. Pan and Q. Yang. A survey on transfer learning. IEEE Transactions on Knowledge and Data Engineering, 22(10):1345-1359, Oct 2010.

[9] C. Prahm, B. Paassen, A. Schulz, B. Hammer, and O. Aszmann. Transfer learning for rapid re-calibration of a myoelectric prosthesis after electrode shift. In J. Ibáñez, J. González-Vargas, J. M. Azorín, M. Akay, and J. L. Pons, editors, Converging Clinical and Engineering Research on Neurorehabilitation II: Proceedings of the 3rd International Conference on NeuroRehabilitation (ICNR2016), October 18-21, 2016, Segovia, Spain, pages 153-157. Springer International Publishing, 2016.

[10] P. Schneider, M. Biehl, and B. Hammer. Adaptive relevance matrices in learning vector quantization. Neural Computation, 21(12):3532-3561, 2009 .

[11] A. Stango, F. Negro, and D. Farina. Spatial correlation of high density emg signals provides features robust to electrode number and shift in pattern recognition for myocontrol. IEEE Transactions on Neural Systems and Rehabilitation Engineering, 23(2):189-198, March 2015.

[12] M. Vidovic, H. J. Hwang, S. Amsuss, J. Hahne, D. Farina, and K. R. Müller. Improving the robustness of myoelectric pattern recognition for upper limb prostheses by covariate shift adaptation. IEEE Transactions on Neural Systems and Rehabilitation Engineering, (99), 2015. 